\title{
Approximate kinetic quasiequilibrium distributions for intense beam propagation through a periodic focusing quadrupole lattice
}

\author{
Edward A. Startsev, Ronald C. Davidson, and Mikhail Dorf \\ Plasma Physics Laboratory, Princeton University, Princeton, New Jersey, 08543
}

(Received 1 September 2009; published 15 June 2010)

\begin{abstract}
The transverse dynamics of an intense charged particle beam propagating through a periodic quadrupole focusing lattice is described by the nonlinear Vlasov-Maxwell system of equations, where the propagation distances play the role of time. To determine matched-beam quasiequilibrium distribution functions, one needs to determine a dynamical invariant for the beam particles moving in the combined applied and self-generated fields. In this paper, a perturbative Hamiltonian transformation method is developed which is an expansion in the particle's vacuum phase advance $\bar{\epsilon} \sim \sigma_{v} / 2 \pi$, treated as a small parameter, which is used to transform away the fast particle orbit oscillations and obtain the average Hamiltonian accurate to order $\bar{\epsilon}^{3}$. The average Hamiltonian is an approximate invariant of the original system, and can be used to determine self-consistent beam quasiequilibrium solutions that are matched to the focusing channel. The equation determining the average self-field potential is derived for general boundary conditions by taking into account the average contribution of the charges induced on the boundary. It is shown for a cylindrical conducting boundary that the average self-field potential acquires an octupole component, which results in the average motion of some beam particles being nonintegrable and their trajectories chaotic. This chaotic behavior of the beam particles may significantly change the nature of the Landau damping (or growth) of collective excitations supported by an intense charged particle beam.
\end{abstract}

DOI: 10.1103/PhysRevSTAB.13.064402

PACS numbers: 29.27.-a, 41.75.-i, 52.59.Sa, 52.59.Tb

\section{INTRODUCTION}

There is growing interest in studying the detailed equilibrium and stability properties of intense charged particle beams for applications to high energy and nuclear physics, high energy density physics research using intense particle beams, and heavy ion beams for inertial fusion energy and warm dense matter applications, etc. [1,2]. In many of the applications, intense charged particle beams have to be transported over long distances through a focusing channel, which provides transverse particle confinement. In a quadrupole focusing channel, the beam particles experience a transverse linear focusing-defocusing force, which is a periodic function of time in the beam frame. This oscillating force provides the necessary focusing only in an average sense [3-7]. For intense charged particle beams, this average focusing force must be strong enough to overcome both thermal and space-charge defocusing of the beam particles.

Identifying regimes for quiescent beam propagation has been one of the main challenges of accelerator research [818]. In particular, the development of systematic approaches that are able to treat self-consistently the applied periodic focusing force and the self-field force of the beam particles simultaneously is very important [19-23]. Several recent investigations [24-26] have used standard Hamiltonian perturbative methods [27-31]. With these methods, one searches for the generating function that relates the old set of canonical phase-space variables to the new canonical set. The new canonical variables are chosen to have a Hamiltonian that is independent of time. In the standard approach, the generating function is a function of the mixed set of variables (old and new). This makes the perturbative analysis moderately complicated. In particular, the analysis in Refs. [24,25] was carried out to third order in the small parameter $\left(\sigma_{v} / 2 \pi\right)^{1 / 2}$, where $\sigma_{v}$ is the vacuum phase advance [5]. The analysis in Ref. [26] was carried out to 5th order, but the authors appeared to have made an error in the iterative procedure, which invalidates the results. An advantage of the approach described in the present analysis is that, instead of using a generating function which is a function of the mixed set of variables, we work with functions that depend exclusively on a new nonoscillating set of variables from the outset. This significantly simplifies the analysis, and allows us to develop an iterative procedure that makes no reference to the generating function in its final form. In this paper, we also make a more consistent ordering of all relevant quantities in the derivation of the canonical transformation. In this new ordering, all quantifies are expanded in the small parameter $\bar{\epsilon} \sim \sigma_{v} / 2 \pi$, which is the square of the small parameter used by previous authors in Refs. [24-26]. As a result, the third-order expansion in the present analysis is equivalent to a sixth-order expansion used in previously developed methods.

For intense charged particle beams, it is important to take special care in determining the self-field potential. The authors of Refs. [25,26] worked directly with 
Poisson's equation, while the author of Ref. [24] worked directly with the Green's function for Poisson's equation. We use the latter approach here because it allows for a simpler treatment, and also allows us to take into account the boundary conditions more easily. The correct treatment of the boundary is very important because the oscillation of charges induced on the conducting boundary produces an important contribution to the average self-field potential, and may significantly change the dynamics of the beam particles.

The organization of this paper is as follows. In Sec. II, the equations describing the nonlinear dynamics of an intense charged particle beam propagating through a quadrupole focusing channel are summarized. The dynamical quantities are normalized, and the small expansion parameter $\epsilon$ is identified. The perturbative Hamiltonian transformation method is developed in Sec. III, and the canonical transformation for arbitrary quadrupole focusing lattice is derived correct to second order in the small parameter $\bar{\epsilon} \sim \sigma_{v} / 2 \pi$. Illustrative examples of the canonical transformation and detailed comparisons with numerical simulations are presented in Sec. IV. Expressions for the average self-field potential are obtained in Sec. V. Finally, the key results and conclusions are summarized in Sec. VI.

\section{THEORETICAL MODEL}

The transverse dynamics of a coasting intense charged particle beam can be described by the nonlinear VlasovPoisson system of equations for the beam distribution function $f\left(x, y, p_{x}, p_{y}, s\right)$ and the normalized self-field potential $\Psi(x, y, s)$. Here $s=v_{b} t$ is the longitudinal coordinate, where $v_{b}=$ const is the directed beam velocity. In what follows, we use index notations where $(x, y) \equiv$ $\left(x^{1}, x^{2}\right)$, and $\left(p_{x}, p_{y}\right) \equiv\left(p^{1}, p^{2}\right)$. For simplicity, we also suppress variable indices inside of function definitions, i.e., we employ the notation $f\left(x^{1}, x^{2}, p^{1}, p^{2}, s\right) \equiv f(x, p, s)$.

The distribution function $f(x, p, s)$ satisfies the nonlinear Vlasov equation [5],

$$
\frac{d f}{d s}=\frac{\partial f}{\partial s}+\sum_{\alpha=1}^{2} \frac{d x^{\alpha}}{d s} \frac{\partial f}{\partial x^{\alpha}}+\sum_{\alpha=1}^{2} \frac{d p^{\alpha}}{d s} \frac{\partial f}{\partial p^{\alpha}}=0,
$$

where

$$
\frac{d x^{\alpha}}{d s}=\frac{\partial H}{\partial p^{\alpha}}, \quad \frac{d p^{\alpha}}{d s}=-\frac{\partial H}{\partial x^{\alpha}}
$$

are the particle equations of motion, and $\alpha=1,2$ refer to the transverse phase-space variables $\left(x, y, p_{x}, p_{y}\right)$. The Hamiltonian $H(x, p, s)$ describes the particle motion in a force field that is the sum of a linear, externally applied, transverse focusing force with components $F_{\text {foc }}^{\alpha}=$ $-\kappa(s) \eta^{\alpha} x^{\alpha}$, where $\kappa(s)$ is the focusing field strength, $\eta^{1}=1, \eta^{2}=-1$, and the normalized self-field potential $\Psi(x, s)$ is calculated self-consistently using Poisson's equation. The Hamiltonian $H(x, p, s)$ is defined by

$$
H(x, p, s)=\sum_{\alpha=1}^{2} \frac{p^{\alpha} p^{\alpha}}{2}+\sum_{\alpha=1}^{2} \kappa(s) \frac{\eta^{\alpha} x^{\alpha} x^{\alpha}}{2}+\Psi(x, s),
$$

where $\kappa(s)$ varies periodically as a function of axial coordinate $s$ according to $\kappa(s)=\kappa(s+S)$, and $S=$ const is the lattice period. In this paper, we consider lattice functions that satisfy the condition $\int_{s}^{s+S} d \bar{s} \kappa(\bar{s})=0$ and have odd half-period symmetry $\kappa(s)=-\kappa(s+S / 2)$. Poisson's equation for the normalized self-field potential $\Psi(x, s)$ is given by

$$
\nabla_{\perp}^{2} \Psi \equiv \sum_{\alpha=1}^{2} \frac{\partial}{\partial x^{\alpha}} \frac{\partial}{\partial x^{\alpha}} \Psi=-\frac{2 \pi A}{N} \int f(x, p, s) d p^{1} d p^{2},
$$

where $N=\int d x^{1} d x^{2} d p^{1} d p^{2} f(x, p, s)$ is the number line density of the beam particles, $A=2 q^{2} N / m_{b} v_{b}^{2} \gamma_{b}^{3}$ is the beam self-field perveance, $m_{b}$ and $q$ are the particle mass and charge, respectively, and $\gamma_{b}=\left(1-v_{b}^{2} / c^{2}\right)^{-1 / 2}$ is the relativistic mass factor.

It is convenient to introduce the dimensionless renormalized variables $\bar{x}=x / a, \bar{s}=s / S, \bar{\kappa}(\bar{s})=\kappa(s) / \kappa_{0}, \bar{p}=$ $p /\left(a \kappa_{0} S\right)$, and $\bar{f}=(f / N) a^{4}\left(\kappa_{0} S\right)^{2}$, where $S$ is the period of the applied focusing lattice, $a$ is the characteristic transverse beam dimension, and $\kappa_{0}$ is the characteristic value of the lattice function $\kappa(s)$. Equations (1) and (2) maintain the same form in normalized variables, whereas the normalized Hamiltonian $\bar{H}$ takes the form

$$
\begin{aligned}
\bar{H}(\bar{x}, \bar{p}, \bar{s})= & \bar{\kappa}(\bar{s}) \frac{\left[\eta^{\alpha} \bar{x}^{\alpha} \bar{x}^{\alpha}\right]}{2} \\
& +\epsilon\left\{\frac{\left[\bar{p}^{\alpha} \bar{p}^{\alpha}\right]}{2}+\int L\left(\bar{x}, \bar{x}^{\prime}\right) \bar{f}\left(\bar{x}^{\prime}, \bar{p}^{\prime}, \bar{s}\right) D \bar{x}^{\prime} D \bar{p}^{\prime}\right\} .
\end{aligned}
$$

For simplicity, we adopt a square-bracket notation for summations. e.g., $\left[x^{\alpha} x^{\alpha}\right] \equiv \sum_{\alpha=1}^{2} x^{\alpha} x^{\alpha}$. Moreover, for multidimensional integrals, we adopt the notation $\int d x_{1} d x_{2} Z=\int D x Z$. In Eq. (5), $\epsilon$ is defined by $\epsilon \equiv$ $S^{2} \kappa_{0}$, and the Green's function $L\left(\bar{x}, \bar{x}^{\prime}\right)$ satisfies the equation

$$
\left[\frac{\partial}{\partial \bar{x}^{\alpha}} \frac{\partial}{\partial \bar{x}^{\alpha}}\right] L\left(\bar{x}, \bar{x}^{\prime}\right)=-s_{b} \delta\left(\bar{x}-\bar{x}^{\prime}\right) .
$$

Here, $s_{b}=2 \pi A /\left(\kappa_{0} S\right)^{2} a^{2}=\left(4 \pi q^{2} N / m_{b} a^{2} \gamma_{b}^{3}\right) /\left(\kappa_{0} S v_{b}\right)^{2}$ is a dimensionless measure of the beam space-charge intensity. For a beam transversely confined by the external focusing lattice, the characteristic maximum value of normalized intensity $s_{b}$ is $\left(s_{b}\right)^{\max } \sim 1$ [5]. In Eq. (5), the function $\bar{f}$ is normalized according to $\int d \bar{x} d \bar{p} \bar{f}=1$. In what follows, we assume that all terms inside the curly brackets in Eq. (5) are of the same order. 


\section{PERTURBATIVE HAMILTONIAN TRANSFORMATION METHOD}

In what follows, we drop the bar notation over the normalized variables. To determine the matched quasiequilibrium solutions to the Vlasov equation (1), we search for a time-dependent canonical transformation of the form $[5,24-26]\left(x^{\alpha}, p^{\alpha}, H, s\right) \rightarrow\left(Q^{\alpha}, P^{\alpha}, K, s\right)$, where

$$
x^{\alpha}=x^{\alpha}(Q, P, s), \quad p^{\alpha}=p^{\alpha}(Q, P, s),
$$

with time-independent transformed Hamiltonian $K(Q, P)$. For every canonical transformation there is a function $\tilde{S}$ that satisfies the differential relation [27-30]

$$
\left[p^{\alpha} d x^{\alpha}\right]-H d s=d \tilde{S}+\left[P^{\alpha} d Q^{\alpha}\right]-K d s .
$$

It is convenient to search for a function $\tilde{S}$ of the form $\tilde{S}=$ $U+\left[p_{0}(Q, P, s)^{\alpha}(x-Q)^{\alpha}\right]$, where $U(Q, P, s)$ and $p_{0}(Q, P, s)$ are functions of the new phase-space variables. The relationships between the old and new set of phasespace coordinates are obtained from Eq. (8) by equating coefficients in front of the differentials of independent variables $\left(d P^{\alpha}, d Q^{\alpha}, d s\right)$, and can be expressed as

$$
\begin{aligned}
{\left[(x-Q)^{\alpha} \frac{\partial p_{0}^{\alpha}}{\partial P^{\beta}}\right]=} & {\left[\left(p-p_{0}\right)^{\alpha} \frac{\partial(x-Q)^{\alpha}}{\partial P^{\beta}}\right]-\frac{\partial U}{\partial P^{\beta}}, } \\
(p-P)^{\beta}= & -\left[\left(p-p_{0}\right)^{\alpha} \frac{\partial(x-Q)^{\alpha}}{\partial Q^{\beta}}\right] \\
& +\frac{\partial U}{\partial Q^{\beta}}+\left[(x-Q)^{\alpha} \frac{\partial p_{0}^{\alpha}}{\partial Q^{\beta}}\right], \\
K-H= & -\left[\left(p-p_{0}\right)^{\alpha} \frac{\partial(x-Q)^{\alpha}}{\partial s}\right]+\frac{\partial U}{\partial s} \\
& +\left[(x-Q)^{\alpha} \frac{\partial p_{0}^{\alpha}}{\partial s}\right] .
\end{aligned}
$$

The canonical transformation procedure outlined by Eqs. (7)-(9) is slightly different from the standard approach. In the standard approach, the generating function $\tilde{S}$ is taken to be a function of the mixed set of variables (old and new). This makes the perturbative analysis moderately complicated [24-26] relative to the present approach. An advantage of the approach described in the present analysis is that, instead of using a generating function which is a function of the mixed set of variables, we work with functions that depend exclusively on the new nonoscillating set of variables $\left(Q^{\alpha}, P^{\alpha}\right)$ from the outset. This significantly simplifies the analysis, and allows us to develop an iterative procedure that makes no reference to the generating function in its final form.

The distribution function in the new coordinates $F(Q, P, s)$ is related to the distribution function in the old coordinates $f(x, p, s)$ by

$$
F(Q, P, s) D Q D P=f(x, p, s) D x D p .
$$

Equation (10) expresses particle conservation in the phase-space volume $D x D p$ under the transformation given by Eq. (7). For a canonical transformation, the phase-space volume is conserved according to $D x D p=D Q D P$, and therefore $F(P, Q, s)=f[x(Q, P, s), p(Q, P, s), s]$. The distribution function in the new variables satisfies the Vlasov equation

$$
\frac{d F}{d s}=0 .
$$

For a time-independent Hamiltonian, there exists a trivial solution to the Vlasov equation (11), given by $F=$ $G[K(Q, P)]$ for arbitrary function $G$. The periodic focusing solution to the original Vlasov equation (1) can be determined by inverting Eq. (7) according to $f(x, p, s)=$ $G\left\{K_{G}\left[Q_{G}(x, p, s), P_{G}(x, p, s)\right]\right\}$. Here, the subscript $G$ denotes the implicit dependence of the solution on the choice of the function $G$. For solutions of this form, we can use Eq. (10) to express the original Hamiltonian in Eq. (5) as

$$
\begin{aligned}
H(x, p, s)= & \frac{\kappa(s)\left[\eta^{\alpha} x^{\alpha} x^{\alpha}\right]}{2}+\epsilon\left\{\frac{\left[p^{\alpha} p^{\alpha}\right]}{2}\right. \\
& \left.+\int L[x, x(\bar{Q}, \bar{P}, s)] G[K(\bar{Q}, \bar{P})] D \bar{Q} D \bar{P}\right\} .
\end{aligned}
$$

Equations (7) and (9) can be solved iteratively in terms of the small parameter $\epsilon=\kappa_{0} S^{2}<1$. Specifically, we express

$$
\begin{aligned}
p & =p_{0}(Q, P, s)+\sum_{n=1} \epsilon^{n} p_{n}, \\
x & =Q+\sum_{n=1} \epsilon^{n} x_{n}, \\
U & =U_{0}(Q, P, s)+\sum_{n=1} \epsilon^{n} U_{n}, \\
K & =K_{0}(Q, P, s)+\sum_{n=1} \epsilon^{n} K_{n},
\end{aligned}
$$

where $p_{n}(Q, P, s), x_{n}(Q, P, s), U_{n}(Q, P, s)$, and $K_{n}(Q, P, s)$ $(n=0,1,2, \ldots)$ are functions to be determined from the iterative procedure. Using Eq. (13), we expand the Hamiltonian $H$ in Eq. (12) according to

$$
H(x, p, s)=\sum_{n=0} \epsilon^{n} H_{n}(Q, P, s) .
$$

Substituting the expansions [Eqs. (13) and (14)] into Eqs. (9), we obtain

$$
\begin{aligned}
& H_{0}(Q, P, s)=\frac{\kappa(s)\left[\eta^{\alpha} Q^{\alpha} Q^{\alpha}\right]}{2}, \\
& p_{0}^{\beta}=P^{\beta}+\frac{\partial U_{0}}{\partial Q^{\beta}}, \\
& \frac{\partial U_{0}}{\partial P^{\beta}}=0, \\
& K_{0}-H_{0}=\frac{\partial U_{0}}{\partial s} .
\end{aligned}
$$

From Eq. (16), we obtain 


$$
K_{0}=\left\langle H_{0}\right\rangle=\langle\kappa\rangle \frac{\left[\eta^{\alpha} Q^{\alpha} Q^{\alpha}\right]}{2}=0
$$

and

$$
\begin{gathered}
U_{0}=-\kappa^{(1)} \frac{\left[\eta^{\alpha} Q^{\alpha} Q^{\alpha}\right]}{2}, \\
p_{0}^{\beta}=P^{\beta}-\kappa^{(1)} \eta^{\beta} Q^{\beta},
\end{gathered}
$$

where $\langle A\rangle \equiv(1 / S) \int_{S}^{s+S} d \bar{s} A(\bar{s})$, and $\langle\langle A\rangle\rangle \equiv A-\langle A\rangle$. Here, we also introduce the notation $A^{(0)} \equiv\langle\langle A\rangle\rangle$ and

$$
A^{(n)} \equiv\left\langle\left\langle\int d s A^{(n-1)}\right\rangle\right\rangle
$$

for $n \geq 1$. If what follows, we will use properties that follow from the definition in Eq. (20), including

$$
A(s)=\langle A\rangle+\left[\frac{d A}{d s}\right]^{(1)}=\langle A\rangle+\frac{d A^{(1)}}{d s},
$$

valid for any periodic function $A(s)=A(s+S)$, and

$$
\begin{gathered}
\frac{d A^{(n+1)}}{d s}=A^{(n)}, \\
\left\langle A^{(k)} B^{(n)}\right\rangle=-\left\langle A^{(k-1)} B^{(n+1)}\right\rangle=-\left\langle A^{(k+1)} B^{(n-1)}\right\rangle, \\
{\left[A^{(k)} B^{(n)}\right]^{(1)}+\left[A^{(k+1)} B^{(n-1)}\right]^{(1)}=\left\langle\left\langle A^{(k+1)} B^{(n)}\right\rangle\right\rangle .}
\end{gathered}
$$

Substituting the expansions [Eqs. (13) and (14)] into Eqs. (9), we obtain

$$
\begin{gathered}
K_{n}=H_{n}+\frac{\partial U_{n}}{\partial s}-\sum_{l=1}^{n-1}\left[p_{n-l}^{\alpha} \frac{\partial x_{l}^{\alpha}}{\partial s}\right]+\left[x_{n}^{\alpha} \frac{\partial p_{0}^{\alpha}}{\partial s}\right], \\
x_{n}^{\beta}=-\frac{\partial U_{n}}{\partial P^{\beta}}+\sum_{l=1}^{n-1}\left[p_{n-l}^{\alpha} \frac{\partial x_{l}^{\alpha}}{\partial P^{\beta}}\right] ; \\
p_{n}^{\beta}=\frac{\partial U_{n}}{\partial Q^{\beta}}-\sum_{l=1}^{n-1}\left[p_{n-l}^{\alpha} \frac{\partial x_{l}^{\alpha}}{\partial Q^{\beta}}\right]+\left[x_{n}^{\alpha} \frac{\partial p_{0}^{\alpha}}{\partial Q^{\beta}}\right],
\end{gathered}
$$

where $n=1,2, \ldots$ From Eq. (25), we obtain

$$
K_{n}=\left\langle H_{n}+\left[x_{n}^{\alpha} \frac{\partial p_{0}^{\alpha}}{\partial s}\right]\right\rangle-\sum_{l=1}^{n-1}\left\langle\left[p_{n-l}^{\alpha} \frac{\partial x_{l}^{\alpha}}{\partial s}\right]\right\rangle
$$

and

$$
U_{n}=\sum_{l=1}^{n-1}\left[p_{n-l}^{\alpha} \frac{\partial x_{l}^{\alpha}}{\partial s}\right]^{(1)}-\left[H_{n}+x_{n}^{\alpha} \frac{\partial p_{0}^{\alpha}}{\partial s}\right]^{(1)}+\bar{U}_{n}(Q, P)
$$

Here, $\bar{U}_{n}$ is an arbitrary function of $P$ and $Q$. We now choose $\bar{U}_{n}$ so that $\left\langle x_{n}\right\rangle=0$. Introducing $Z_{n} \equiv H_{n}-$ $\kappa\left[\eta^{\alpha} x_{n}^{\alpha} Q^{\alpha}\right]$, and substituting Eq. (28) into Eq. (26), we obtain

$$
K_{n}=\left\langle Z_{n}\right\rangle-\sum_{l=1}^{n-1}\left\langle\left[p_{n-l}^{\alpha} \frac{\partial x_{l}^{\alpha}}{\partial s}\right]\right\rangle
$$

$$
\begin{aligned}
x_{n}^{\beta}= & \frac{\partial Z_{n}^{(1)}}{\partial P^{\beta}}+\sum_{l=1}^{n-1}\left[\frac{\partial p_{n-l}^{\alpha}}{\partial s} \frac{\partial x_{l}^{\alpha}}{\partial P^{\beta}}-\frac{\partial p_{n-l}^{\alpha}}{\partial P^{\beta}} \frac{\partial x_{l}^{\alpha}}{\partial s}\right]^{(1)}, \\
p_{n}^{\beta}= & \bar{p}_{n}^{\beta}-\frac{\partial Z_{n}^{(1)}}{\partial Q^{\beta}}-\left\langle\left\langle\kappa^{(1)} \eta^{\beta} x_{n}^{\beta}\right\rangle\right\rangle \\
& -\sum_{l=1}^{n-1}\left[\frac{\partial p_{n-l}^{\alpha}}{\partial s} \frac{\partial x_{l}^{\alpha}}{\partial Q^{\beta}}-\frac{\partial p_{n-l}^{\alpha}}{\partial Q^{\beta}} \frac{\partial x_{l}^{\alpha}}{\partial s}\right]^{(1)},
\end{aligned}
$$

where the average value $\bar{p}_{n}$ satisfies

$$
\begin{aligned}
\frac{\partial \bar{p}_{n}^{\gamma}}{\partial P^{\beta}}= & \left\langle\sum_{l=1}^{n-1}\left[\frac{\partial p_{n-l}^{\alpha}}{\partial Q^{\gamma}} \frac{\partial x_{l}^{\alpha}}{\partial P^{\beta}}-\frac{\partial p_{n-l}^{\alpha}}{\partial P^{\beta}} \frac{\partial x_{l}^{\alpha}}{\partial Q^{\gamma}}\right]\right\rangle \\
& -\frac{\partial}{\partial P^{\beta}}\left\langle\eta^{\gamma} x_{n}^{\gamma} \kappa^{(1)}\right\rangle .
\end{aligned}
$$

For the Hamiltonian function $H(x, p, s)$ [Eq. (12)] the functions $Z_{n}$ depend only on $p_{k}$ and $x_{k}$, with $k<n$. Therefore, Eqs. (29)-(31) provide an iterative procedure which can be used to determine the canonical transformation in Eq. (7), and the new time-independent Hamiltonian $K(P, Q)$ as implicit functions of the equilibrium distribution function $G$. In this paper we present results valid up to second order in the small parameter $\epsilon$ for the canonical transformation in Eq. (13), and up to third order in $\epsilon$ for the average Hamiltonian $K$. Because $K_{0}=0$, the average Hamiltonian $K$ has the form $K=\epsilon\left(K_{1}+\epsilon K_{2}+\epsilon^{2} K_{3}+\right.$ $\cdots)$. The $\epsilon$ in front of the bracket renormalizes the time scale, so that the average dynamics occurs on the slow time scale $Q=Q(\epsilon s)$ and $P=P(\epsilon s)$. Therefore, to determine the trajectories $x(s)$ and $p(s)$ valid to second order in $\epsilon$, we need to determine the average Hamiltonian $K$ valid up to the third order in $\epsilon$.

Omitting algebraic details, it is straightforward to show that the canonical transformation takes the form

$x^{\alpha}=Q^{\alpha}-\epsilon \kappa^{(2)} \eta^{\alpha} Q^{\alpha}+\epsilon^{2}\left\{2 \kappa^{(3)} \eta^{\alpha} P^{\alpha}+\left(\kappa \kappa^{(2)}\right)^{(2)} Q^{\alpha}\right\}$,

and

$$
\begin{aligned}
p^{\alpha}= & \left\{P^{\alpha}-\kappa^{(1)} \eta^{\alpha} Q^{\alpha}\right\}+\epsilon\left\{\kappa^{(2)} \eta^{\alpha} P^{\alpha}+\left(\kappa \kappa^{(2)}\right)^{(1)} Q^{\alpha}\right\}+\epsilon^{2}\left\{\kappa^{(3)} \int D \bar{Q} D \bar{P} G \frac{\partial}{\partial Q^{\alpha}}\left[\eta^{\beta} Q^{\beta} \frac{\partial L}{\partial Q^{\beta}}+\eta^{\beta} \bar{Q}^{\beta} \frac{\partial L}{\partial \bar{Q}^{\beta}}\right]\right. \\
& \left.+\left[3\left\langle\left(\kappa^{(2)}\right)^{2}\right\rangle-2\left(\kappa \kappa^{(3)}\right)^{(1)}-\left(\kappa \kappa^{(2)}\right)^{(2)}\right] P^{\alpha}+\left\{\kappa^{(3)}\left\langle\left(\kappa^{(1)}\right)^{2}\right\rangle-\left[\kappa\left(\kappa \kappa^{(2)}\right)^{(2)}\right]^{(1)}\right\} \eta^{\alpha} Q^{\alpha}\right\} .
\end{aligned}
$$


Moreover, the inverse transformation is given by

$$
Q^{\alpha}=x^{\alpha}+\epsilon \kappa^{(2)} \eta^{\alpha} x^{\alpha}+\epsilon^{2}\left\{-2 \kappa^{(3)} \eta^{\alpha} p^{\alpha}+\left[3\left\langle\left(\kappa^{(2)}\right)^{2}\right\rangle-2\left(\kappa \kappa^{(3)}\right)^{(1)}-\left(\kappa \kappa^{(2)}\right)^{(2)}\right] x^{\alpha}\right\},
$$

and

$$
\begin{aligned}
P^{\alpha}= & \left\{p^{\alpha}+\kappa^{(1)} \eta^{\alpha} x^{\alpha}\right\}-\epsilon\left\{\kappa^{(2)} \eta^{\alpha} p^{\alpha}+\left(\kappa \kappa^{(2)}\right)^{(1)} x^{\alpha}\right\}-\epsilon^{2}\left\{\kappa^{(3)} \int D \bar{x} D \bar{p} G \frac{\partial}{\partial x^{\alpha}}\left[\eta^{\beta} x^{\beta} \frac{\partial L}{\partial x^{\beta}}+\eta^{\beta} \bar{x}^{\beta} \frac{\partial L}{\partial \bar{x}^{\beta}}\right]\right. \\
& \left.-\left(\kappa \kappa^{(2)}\right)^{(2)} p^{\alpha}+\left\{\kappa^{(3)}\left\langle\left(\kappa^{(1)}\right)^{2}\right\rangle-\left[\kappa\left(\kappa \kappa^{(2)}\right)^{(2)}\right]^{(1)}\right\} \eta^{\alpha} x^{\alpha}\right\} .
\end{aligned}
$$

The new time-independent Hamiltonian is then determined to be (correct to the third order in $\epsilon$ )

$$
\begin{aligned}
K= & \epsilon\left\{\frac{\left[P^{\alpha} P^{\alpha}\right]}{2}\left[1+3 \epsilon^{2}\left\langle\left(\kappa^{(2)}\right)^{2}\right\rangle\right]+\frac{\left[Q^{\alpha} Q^{\alpha}\right]}{2}\left\{\left\langle\left(\kappa^{(1)}\right)^{2}\right\rangle+\epsilon\left\langle\kappa\left(\kappa^{(2)}\right)^{2}\right\rangle+\epsilon^{2}\left\langle\left[\left(\kappa \kappa^{(2)}\right)^{(1)}\right]^{2}\right\rangle\right\}\right. \\
& +\int D \bar{Q} D \bar{P} G(\bar{K})\left(L(Q, \bar{Q})+\epsilon^{2} \frac{\left\langle\left(\kappa^{(2)}\right)^{2}\right\rangle}{2}\left[\eta^{\alpha} \eta^{\beta} Q^{\alpha} Q^{\beta} \frac{\partial^{2} L}{\partial Q^{\alpha} \partial Q^{\beta}}+2 \eta^{\alpha} \eta^{\beta} Q^{\alpha} \bar{Q}^{\beta} \frac{\partial^{2} L}{\partial Q^{\alpha} \partial \bar{Q}^{\beta}}\right.\right. \\
& \left.\left.\left.+\eta^{\alpha} \eta^{\beta} \bar{Q}^{\alpha} \bar{Q}^{\beta} \frac{\partial^{2} L}{\partial \bar{Q}^{\alpha} \partial \bar{Q}^{\beta}}\right]\right)\right\} .
\end{aligned}
$$

For a periodic lattice with $o d d$ half-lattice-period symmetry, $\kappa(s)=-\kappa(s+S / 2)$, the term $\left\langle\kappa\left(\kappa^{(2)}\right)^{2}\right\rangle$ occurring in Eq. (36) is identically zero, i.e., $\left\langle\kappa\left(\kappa^{(2)}\right)^{2}\right\rangle \equiv 0$, and therefore the normalized average Hamiltonian $K / \epsilon$ is expanded in a series of even powers $\left(\epsilon^{2 k}\right)$ of the small parameter $\epsilon$.

Using Eq. (36), we obtain the approximate expression for the square of the vacuum phase advance $\sigma_{v}^{2}$ valid up to fourth order in small parameter $\epsilon$, i.e.,

$$
\begin{aligned}
\sigma_{v}^{2}= & S^{2} \epsilon^{2}\left\{1+3 \epsilon^{2}\left\langle\left(\kappa^{(2)}\right)^{2}\right\rangle\right\}\left\langle\left\langle\left(\kappa^{(1)}\right)^{2}\right\rangle+\epsilon\left\langle\kappa\left(\kappa^{(2)}\right)^{2}\right\rangle\right. \\
& \left.+\epsilon^{2}\left\langle\left[\left(\kappa \kappa^{(2)}\right)^{(1)}\right]^{2}\right\rangle\right\} .
\end{aligned}
$$

Note from Eqs. (32)-(37) and the definitions in Eq. (20) that the actual expansion parameter in Eqs. (32)-(37) is not $\epsilon$ but rather $\bar{\epsilon} \equiv \epsilon\left[\left\langle\left(\kappa^{(1)}\right)^{2}\right\rangle\right]^{1 / 2} \sim \sigma_{v} /(2 \pi)$. For a lattice with small filling factor $\delta \sim T / S \ll 1$, when the focusing elements occupy a distance $2 T$ which is a small portion of the lattice period $S$, the correction $\left[\left\langle\left(\kappa^{(1)}\right)^{2}\right\rangle\right]^{1 / 2} \sim \delta$ can be quite important. For such lattices the theory presented in this paper still applies even if $\epsilon>1$, provided the condition $\epsilon \delta \ll 1$ still holds. It can be easily shown that for intense beams with normalized intensity $s_{b} \lesssim 1$ when the self-field part of the average Hamiltonian $K$ is of the same order as the external focusing part, which is in turn of the same order as the kinetic part

$$
\int D \bar{Q} D \bar{P} G(\bar{K}) L(Q, \bar{Q}) \sim \frac{\left[Q^{\alpha} Q^{\alpha}\right]}{2}\left\langle\left(\kappa^{(1)}\right)^{2}\right\rangle \sim \frac{\left[P^{\alpha} P^{\alpha}\right]}{2},
$$

then the self-field terms in the expressions for the canonical transformation in Eq. (32)-(35) have an order which is consistent with expansion in the small parameter $\sigma_{v} /(2 \pi) \sim \epsilon \delta$. Note that for very intense beams with $s_{b} \lesssim$ 1 , the external focusing force is reduced by the repulsive self-field force, and one expects the expansion parameter to be proportional to the depressed phase advance $\sigma / 2 \pi$
[5-7]. However, for moderately intense beams, the depressed phase advance for transverse particle oscillations with average Hamiltonian $K$ given by Eq. (36) is of the same order as the vacuum phase advance, i.e., $\sigma \sim \sigma_{v}$. The case of extremely intense beams, when the depressed phase advance is much smaller than vacuum phase advance $\sigma \ll$ $\sigma_{v}$, requires special consideration and is not analyzed here.

\section{ILLUSTRATIVE APPLICATIONS}

As a specific application, in this section we examine the canonical transformation in Eq. (7), valid up to second order in the small parameter $\epsilon$, for the intense beam system with Hamiltonian given by Eq. (12), for the specific choice of sinusoidal lattice function $\kappa(s)=\bar{\kappa} \sin (k s)$ with lattice period $S=2 \pi / k$. The new time-independent Hamiltonian is then determined to be

$$
\begin{aligned}
K= & \epsilon\left[\frac{\left[P^{\alpha} P^{\alpha}\right]}{2}\left(1+\epsilon^{2} \frac{3 \bar{\kappa}^{2}}{2 k^{4}}\right)+\frac{\bar{\kappa}^{2}}{2 k^{2}} \frac{\left[Q^{\alpha} Q^{\alpha}\right]}{2}\left(1+\epsilon^{2} \frac{\bar{\kappa}^{2}}{16 k^{4}}\right)\right. \\
& +\int D \bar{Q} D \bar{P} G(\bar{K})\left(L(Q, \bar{Q})+\epsilon^{2} \frac{\bar{\kappa}^{2}}{4 k^{4}}\right. \\
& \times\left[\eta^{\alpha} \eta^{\beta} Q^{\alpha} Q^{\beta} \frac{\partial^{2} L}{\partial Q^{\alpha} \partial Q^{\beta}}+2 \eta^{\alpha} \eta^{\beta} Q^{\alpha} \bar{Q}^{\beta} \frac{\partial^{2} L}{\partial Q^{\alpha} \partial \bar{Q}^{\beta}}\right. \\
& \left.\left.\left.+\eta^{\alpha} \eta^{\beta} \bar{Q}^{\alpha} \bar{Q}^{\beta} \frac{\partial^{2} L}{\partial \bar{Q}^{\alpha} \partial \bar{Q}^{\beta}}\right]\right)\right\} .
\end{aligned}
$$

From Eq. (39), we obtain for the vacuum phase advance

$$
\left(\frac{\sigma_{v}}{2 \pi}\right)^{2}=\epsilon^{2} \frac{\bar{\kappa}^{2}}{2 k^{4}}\left(1+\epsilon^{2} \frac{3 \bar{\kappa}^{2}}{2 k^{4}}\right)\left(1+\epsilon^{2} \frac{\bar{\kappa}^{2}}{16 k^{4}}\right)
$$

or equivalently,

$$
\left(\frac{\sigma_{v}}{2 \pi}\right)^{2}=\left(\frac{\sigma_{v}^{0}}{2 \pi}\right)^{2}\left[1+3\left(\frac{\sigma_{v}^{0}}{2 \pi}\right)^{2}\right]\left[1+\frac{1}{8}\left(\frac{\sigma_{v}^{0}}{2 \pi}\right)^{2}\right],
$$


where

$$
\left(\frac{\sigma_{v}^{0}}{2 \pi}\right)=\frac{\epsilon}{\sqrt{2}}\left[\frac{\bar{\kappa} S^{2}}{(2 \pi)^{2}}\right] .
$$

Furthermore, the detailed expressions for the canonical transformation are given by

$$
\begin{aligned}
x^{\alpha}= & Q^{\alpha}+\epsilon\left\{\frac{\bar{\kappa}}{k^{2}} \eta^{\alpha} Q^{\alpha} \sin (k s)\right\} \\
& +\epsilon^{2}\left\{2 \frac{\bar{\kappa}}{k^{3}} \eta^{\alpha} P^{\alpha} \cos (k s)-\frac{\bar{\kappa}^{2}}{8 k^{4}} Q^{\alpha} \cos (2 k s)\right\},
\end{aligned}
$$

and

$$
\begin{aligned}
p^{\alpha}= & \left\{P^{\alpha}+\frac{\bar{\kappa}}{k} \eta^{\alpha} Q^{\alpha} \cos (k s)\right\}-\epsilon\left\{\frac{\bar{\kappa}}{k^{2}} \eta^{\alpha} P^{\alpha} \sin (k s)-\frac{\bar{\kappa}^{2}}{4 k^{3}} Q^{\alpha} \sin (2 k s)\right\} \\
& +\epsilon^{2}\left\{\frac{\bar{\kappa}}{k^{3}} \cos (k s) \int D \bar{Q} D \bar{P} G \frac{\partial}{\partial Q^{\alpha}}\left[\eta^{\beta} Q^{\beta} \frac{\partial L}{\partial Q^{\beta}}+\eta^{\beta} \bar{Q}^{\beta} \frac{\partial L}{\partial \bar{Q}^{\beta}}\right]+\frac{\bar{\kappa}^{2}}{8 k^{4}} P^{\alpha}[12+5 \cos (2 k s)]\right. \\
& \left.+\frac{\bar{\kappa}^{3}}{16 k^{5}} \eta^{\alpha} Q^{\alpha}\left(9 \cos (k s)-\frac{1}{3} \cos (3 k s)\right)\right\} .
\end{aligned}
$$

Furthermore, the inverse transformation is given by

$$
Q^{\alpha}=x^{\alpha}-\epsilon\left\{\frac{\bar{\kappa}}{k^{2}} \eta^{\alpha} x^{\alpha} \sin (k s)\right\}-\epsilon^{2}\left\{2 \frac{\bar{\kappa}}{k^{3}} \eta^{\alpha} p^{\alpha} \cos (k s)-\frac{\bar{\kappa}^{2}}{8 k^{4}}[12+5 \cos (2 k s)] x^{\alpha}\right\},
$$

and

$$
\begin{aligned}
P^{\alpha}= & \left\{p^{\alpha}-\frac{\bar{\kappa}}{k} \eta^{\alpha} x^{\alpha} \cos (k s)\right\}+\epsilon\left\{\frac{\bar{\kappa}}{k^{2}} \eta^{\alpha} p^{\alpha} \sin (k s)-\frac{\bar{\kappa}^{2}}{4 k^{3}} x^{\alpha} \sin (2 k s)\right\} \\
& -\epsilon^{2}\left\{\frac{\bar{\kappa}}{k^{3}} \cos (k s) \int D \bar{x} D \bar{p} G \frac{\partial}{\partial x^{\alpha}}\left[\eta^{\beta} x^{\beta} \frac{\partial L}{\partial x^{\beta}}+\eta^{\beta} \bar{x}^{\beta} \frac{\partial L}{\partial \bar{x}^{\beta}}\right]+\frac{\bar{\kappa}^{2}}{8 k^{4}} p^{\alpha}+\frac{\bar{\kappa}^{3}}{16 k^{5}} \eta^{\alpha} x^{\alpha}\left(9 \cos (k s)-\frac{1}{3} \cos (3 k s)\right)\right\} .
\end{aligned}
$$

Figure 1 shows the differences $\Delta Q(s)=Q(s)-$ $Q_{t r}(x, p, s), \quad \Delta P(s)=P(s)-P_{t r}(x, p, s)$, and $\Delta K / \epsilon=$ $\left[K-K_{t r}(x, p, s)\right] / \epsilon$ plotted as functions of the normalized variable $s / S$ over the interval $s=\left[0,1 / \epsilon^{4}\right]$ for the case of negligible space charge $s_{b} \rightarrow 0$, and for $\bar{\kappa}=k=1, \epsilon=$ $0.1\left(\sigma_{v}=25^{\circ}\right)$. Here, the differences are between the numerical solution of Hamilton's equations of motion for the average Hamiltonian given by Eq. (39) $[Q(s), P(s), K]$, and the solution $\left[Q_{t r}(x, p, s), P_{t r}(x, p, s), K_{t r}(x, p, s)\right]$ obtained by solving numerically the original system of equations with Hamiltonian given by Eq. (12) and using the transformation formulas given by Eqs. (45) and (46). The same initial conditions were used in both cases, i.e., $\quad Q(0)=Q_{t r}(0)=0$ and $P(0)=P_{t r}(0)=[(1+$ $\left.\left.\epsilon^{2} / 16\right) /\left(1+3 \epsilon^{2} / 2\right)\right]^{1 / 2}$. As evident from Fig. 1, the differences $\Delta Q$ and $\Delta P$ grow linearly as $\epsilon^{5} s$ from values of order $\epsilon^{3}$ at time $s / S \sim 1$. Such secular growth is expected since the next-order correction to the phase advance is of order $\epsilon^{5}$. On the other hand, the average energy difference stays constant at its initial value of order $\Delta K / \epsilon \sim \epsilon^{3}$. This is also expected, since $K$ given by Eqs. (39) is an adiabatic invariant for the original system with Hamiltonian in Eq. (12), and therefore is conserved with exponential accuracy, i.e., the value of $(s / S)_{0}$ when the difference in invariant becomes of order its initial value $\delta K / \epsilon \sim$ $\Delta K / \epsilon \sim \epsilon^{3}$ is given by $(s / S)_{0} \sim \exp ($ const $/ \epsilon) / \epsilon^{l}$ for some power index $l$ [29-31].
As a second example, we consider here the periodic step-function lattice specified by

$$
\begin{array}{ll}
\kappa(s)=-\bar{\kappa}, & \text { for }|s-S / 2|<\delta S / 4, \\
\kappa(s)=\bar{\kappa}, & \text { for } 0<s<\delta S / 4 \text { and } S-\delta S / 4<s<S, \\
\kappa(s)=0, & \text { for } \delta S / 4<s<S / 2-\delta S / 4 \text { and } \\
& S / 2+\delta S / 4<s<S-\delta S / 4 .
\end{array}
$$

Here, the constant filling factor $\delta$ lies in the interval $0<$ $\delta<1$, and the coefficients in the average Hamiltonian $K$ in Eq. (39) are given by

$$
\begin{aligned}
\left\langle\left(\kappa^{(1)}\right)^{2}\right\rangle= & \frac{\bar{\kappa}^{2} S^{2} \delta^{2}}{16}\left(1-\frac{2 \delta}{3}\right), \\
\left\langle\left(\kappa^{(2)}\right)^{2}\right\rangle= & \frac{\bar{\kappa}^{2} S^{4} \delta^{2}}{3840}\left(5-5 \delta^{2}+2 \delta^{3}\right), \\
\left\langle\kappa\left(\kappa^{(2)}\right)^{2}\right\rangle= & 0, \\
\left\langle\left[\left(\kappa \kappa^{(2)}\right)^{(1)}\right]^{2}\right\rangle= & \frac{\bar{\kappa}^{4} S^{6} \delta^{4}}{3870720}\left(315-1050 \delta+1337 \delta^{2}\right. \\
& \left.-768 \delta^{3}+168 \delta^{4}\right) .
\end{aligned}
$$

Using Eqs. (37) and (48), we obtain the approximate expression for the square of the vacuum phase advance 

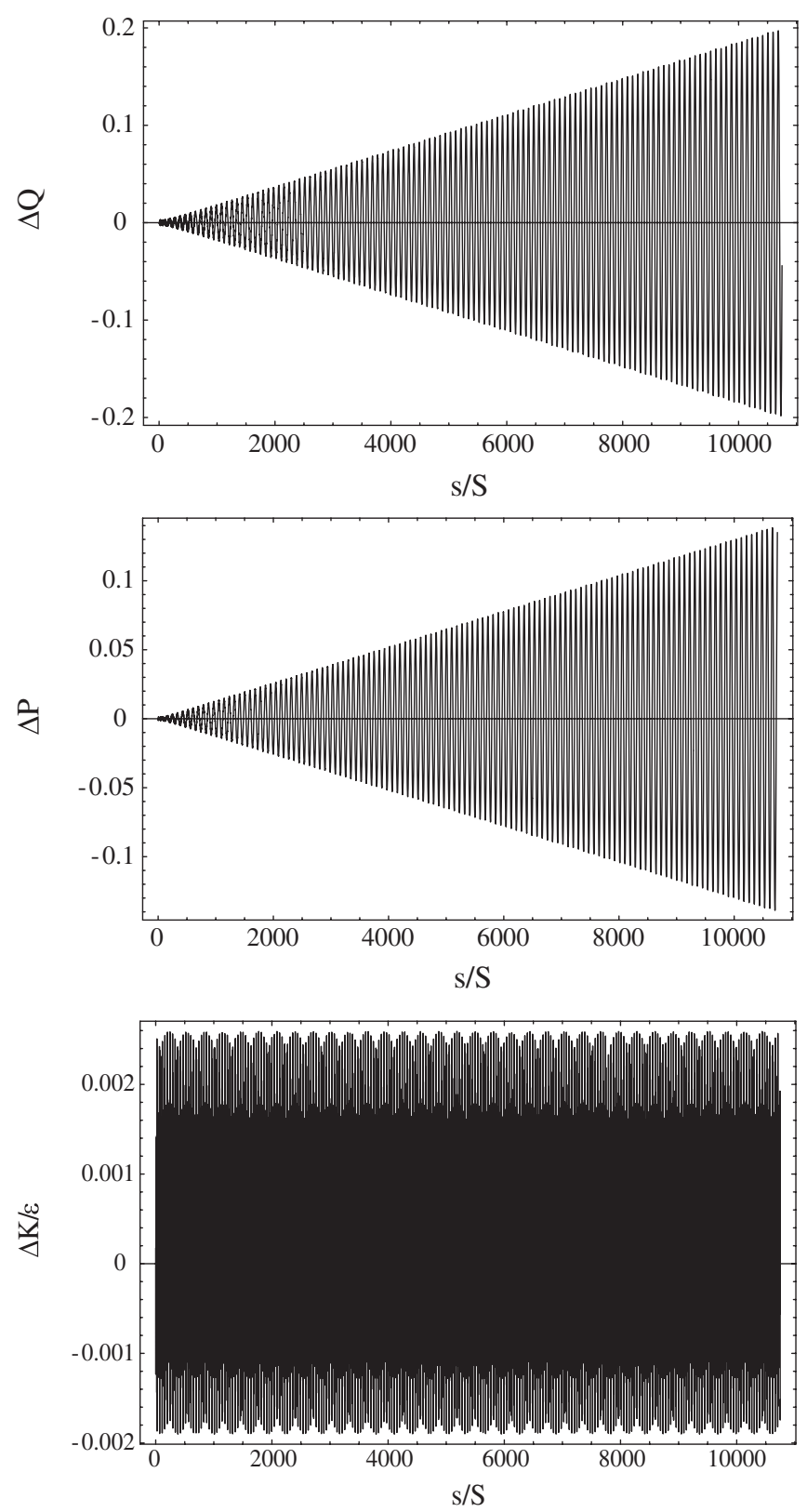

FIG. 1. Plots of $\Delta Q(s)=Q(s)-Q_{t r}(x, p, s), \quad \Delta P(s)=$ $P(s)-P_{t r}(x, p, s)$, and $\Delta K / \epsilon=\left[K-K_{t r}(x, p, s)\right] / \epsilon$ as functions of the normalized variable $s / S$ over the interval $\left[0,1 / \epsilon^{4}\right]$ for the choice of dimensionless parameters $s_{b}=0, \bar{\kappa}=k=1$, $\epsilon=0.1\left(\sigma_{v}=25^{\circ}\right)$.

$$
\begin{aligned}
\sigma_{v}^{2}= & \phi_{0}^{4}\left(1-\frac{2 \delta}{3}\right) \\
& +\phi_{0}^{8} \frac{\left(315-420 \delta+98 \delta^{2}+60 \delta^{3}-21 \delta^{4}\right)}{3780},
\end{aligned}
$$

where $\phi_{0} \equiv\left(\epsilon \bar{\kappa} S^{2} \delta / 4\right)^{1 / 2}$. For the step-function lattice given by Eq. (47), the vacuum phase advance can be calculated exactly $[5,6]$, and is given by

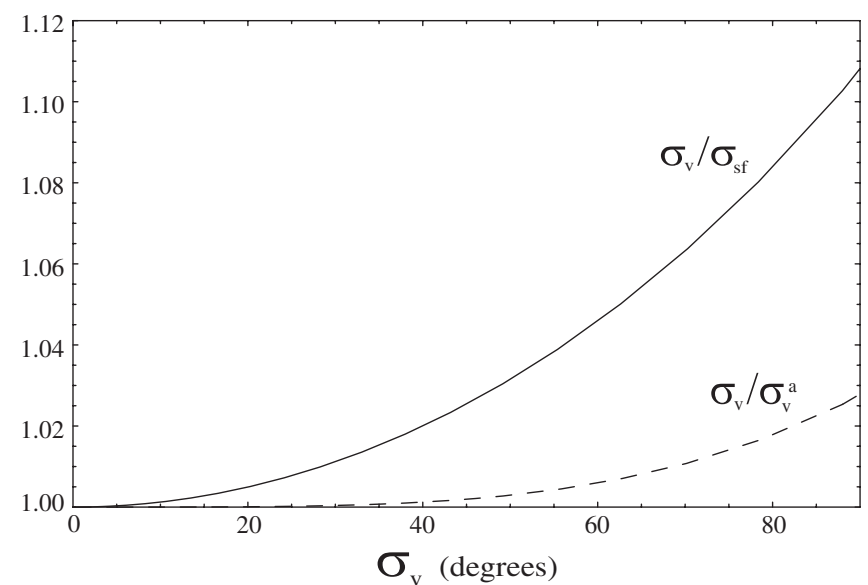

FIG. 2. Plots of the normalized quantities $\sigma_{v} / \sigma_{s f}$ and $\sigma_{v} / \sigma_{v}^{a}$ versus the vacuum phase advance $\sigma_{v}$ for $\delta=1 / 2$.

$$
\begin{aligned}
\cos \left(\sigma_{v}\right)= & \left(\cosh \phi_{1}\right)\left(\cos \phi_{1}-\phi_{2} \sin \phi_{1}\right) \\
& +\phi_{2}\left(\sinh \phi_{1}\right)\left(\cos \phi_{1}-\frac{\phi_{2}}{2} \sin \phi_{1}\right)
\end{aligned}
$$

where $\phi_{1}=\delta^{1 / 2} \phi_{0}$ and $\phi_{2}=\phi_{1}(1-\delta) / \delta$ and $\phi_{0}=$ $\left(\epsilon \bar{\kappa} S^{2} \delta / 4\right)^{1 / 2}$. Taylor expansion of Eq. (50) up to the fourth order in the small parameter $\epsilon$ reproduces exactly the result given in Eq. (49). Shown in Fig. 2 are the plots of $\sigma_{v} / \sigma_{s f}$ and $\sigma_{v} / \sigma_{v}^{a}$ versus $\sigma_{v}$ for $\delta=1 / 2$, where $\sigma_{s f}$ is given by the first term in Eq. (49). Here $\sigma_{v}^{a}$ and $\sigma_{v}$ are obtained from Eqs. (49) and (50), respectively. It is evident from Fig. 2 that the next nonvanishing approximation to the vacuum phase advance $\sigma_{v}^{a}$ given by Eq. (49) is accurate to within $0.6 \%$ for $\sigma_{v} \leq 60^{\circ}$, and is accurate to within $3 \%$ for $\sigma_{v} \leq$ $90^{\circ}$, while the first-order approximation $\sigma_{s f}$ given by the first term in Eq. (49) is only accurate to within 5\% for $\sigma_{v} \leq 60^{\circ}$, and is accurate to within $12 \%$ for $\sigma_{v} \leq 90^{\circ}$.

Note from Eq. (49), as we already mentioned at the end of Sec. III, that the actual expansion parameter for the present theory is $\sigma_{v} / 2 \pi \sim \epsilon \delta \ll 1$, and not simply $\epsilon$. The distinction is important for lattices with small filling factor $\delta \ll 1$. For such lattices the theory presented in this paper still applies even if $\epsilon>1$, provided the condition $\epsilon \delta \ll 1$ still holds.

\section{EVALUATION OF SELF-FIELD POTENTIAL}

The corrections to the average Hamiltonian in Eq. (36) are of two kinds. The corrections to the kinetic energy term and the average applied focusing term give corrections to the average frequency of the particle motion in the applied oscillating field in Eq. (37), whereas the final term gives the corrections to the average self-field potential. The integral form of the self-field potential is inconvenient for numerical calculations because it contains the convolution of the beam density $n(Q)=\int D P G(K)$ with the long-range function $L(Q, \bar{Q})$. The self-field term can be expressed as 
a self-field potential $\bar{\Psi}$ that satisfies the equation

$$
\begin{aligned}
\bar{\Psi}(Q) & =\left\langle\int D \bar{Q} L\left[Q_{\alpha}\left(1+\epsilon \eta^{\alpha} v\right), \bar{Q}_{\alpha}\left(1+\epsilon \eta^{\alpha} v\right)\right] n(\bar{Q})\right\rangle \\
& \equiv\left\langle\Psi_{v}(Q)\right\rangle,
\end{aligned}
$$

where $v \equiv \kappa^{(2)}$. Note that the expansion of the expression in Eq. (51) to second order in terms of the small parameter $\epsilon$ reproduces the self-field term in Eq. (36). This expression for the average self-field potential is general and is valid for the case where the Green's function $L$ satisfies general boundary conditions. In what follows, we make use of the representation given by Eq. (51) to obtain the differential equation for the average potential $\bar{\Psi}(Q)$ for general boundary conditions. In Eq. (51), the Green's function $L$ is chosen to satisfy the boundary conditions for the unaveraged potential in the spacial coordinates $\left(x^{1}, x^{2}\right)$. The boundary conditions for the unaveraged potential in the coordinates $\left(x^{1}, x^{2}\right)$ are introduced in a nontrivial way into the average potential $\bar{\Psi}(Q)$ in the spacial coordinates $\left(Q^{1}, Q^{2}\right)$ through the Green's function $L$ as given by Eq. (51).

By changing the integration variables, $\Psi_{v}(Q)$ in Eq. (51) can be expressed as $\Psi_{v}(Q)=\Phi_{v}\left[Q^{\alpha} /\left(1+\epsilon \eta^{\alpha} v\right]\right.$, where $\Phi_{v}(Q)$ is given by

$$
\Phi_{v}(Q)=\frac{1}{1-\epsilon^{2} v^{2}} \int D \bar{Q} L(Q, \bar{Q}) n\left(\frac{\bar{Q}^{\alpha}}{1+\epsilon \eta^{\alpha} v}\right)
$$

Making use of these definitions, and expanding correct to second order in the small parameter $\epsilon$, we obtain the expression for the average potential:

$$
\begin{aligned}
\bar{\Psi}(Q)= & \left(1+\epsilon^{2}\left\langle v^{2}\right\rangle\right) \phi_{0}+\epsilon^{2}\left\langle v^{2}\right\rangle\left(\phi_{1}-\left[\eta^{\alpha} Q^{\alpha} \frac{\partial}{\partial Q^{\alpha}}\right] \phi_{2}\right. \\
& \left.+\frac{1}{2}\left[\eta^{\alpha} \eta^{\beta} Q^{\alpha} Q^{\beta} \frac{\partial^{2}}{\partial Q^{\alpha} \partial Q^{\beta}}\right] \phi_{0}\right)
\end{aligned}
$$

where the functions $\phi_{0}(Q), \phi_{1}(Q)$, and $\phi_{2}(Q)$ satisfy the Poisson-type equations,

$$
\begin{aligned}
\nabla_{\perp}^{2} \phi_{0}= & -s_{b} n(Q), \\
\nabla_{\perp}^{2} \phi_{1}= & -s_{b}\left(\left[Q^{\alpha} \frac{\partial}{\partial Q^{\alpha}}\right]+\frac{1}{2}\left[\eta^{\alpha} \eta^{\beta} Q^{\alpha} Q^{\beta} \frac{\partial^{2}}{\partial Q^{\alpha} \partial Q^{\beta}}\right]\right) \\
& \times n(Q), \\
\nabla_{\perp}^{2} \phi_{2}= & -s_{b}\left[\eta^{\alpha} Q^{\alpha} \frac{\partial}{\partial Q^{\alpha}}\right] n(Q),
\end{aligned}
$$

where $\nabla_{\perp}^{2} \equiv\left(\partial / \partial Q^{1}\right)\left(\partial / \partial Q^{1}\right)+\left(\partial / \partial Q^{2}\right)\left(\partial / \partial Q^{2}\right)$, and

$$
\nabla_{\perp}^{2} L(Q, \bar{Q})=-s_{b} \delta(Q-\bar{Q})
$$

To solve Eqs. (54), one needs to specify some boundary surface in the coordinate space $\left(Q^{1}, Q^{2}\right)$ and certain boundary conditions on this boundary. It is convenient to designate this boundary surface to be a surface in the coordinate space $\left(Q^{1}, Q^{2}\right)$, where the function $L(Q, \bar{Q})$ satisfies the same boundary conditions as the function $L(x, \bar{x})$ in the coordinate space $\left(x^{1}, x^{2}\right)$. In that case, the boundary conditions for $\phi_{0}(Q), \phi_{1}(Q)$, and $\phi_{2}(Q)$ in Eqs. (54) in the coordinate space $\left(Q^{1}, Q^{2}\right)$ are the same as the boundary conditions for the Green's function $L(Q, \bar{Q})$. Note that this boundary surface in the coordinate space $\left(Q^{1}, Q^{2}\right)$ becomes a surface that oscillates around the boundary surface in the coordinate space $\left(x^{1}, x^{2}\right)$. Because the two surfaces differ, the average potential $\bar{\Psi}(Q)$ in the coordinate space $\left(Q^{1}, Q^{2}\right)$ does not satisfy the same boundary conditions as the unaveraged potential in the coordinate space $\left(x^{1}, x^{2}\right)$, as can be seen from Eq. (53).

Next we introduce the zero-order smooth-focusing potential $\Phi_{0}(Q)$ that satisfies the equilibrium Poisson's equation [5],

$$
\nabla_{\perp}^{2} \Phi_{0}=-s_{b} n_{0}(Q)
$$

with the same boundary conditions as the Green's function $L(Q, \bar{Q})$. Here, $n_{0}(Q) \equiv n\left(Q, \Phi_{0}\right)$. Making use of this definition and Eqs. (53) and (54), we can express the average potential and the particle density correct to second order in $\epsilon$ as

$$
\begin{aligned}
\bar{\Psi} & =\Phi_{0}+\epsilon^{2}\left\langle v^{2}\right\rangle \bar{\Psi}_{1}, \\
n[Q, \bar{\Psi}(Q)] & =n_{0}+\epsilon^{2}\left\langle v^{2}\right\rangle n_{0}^{\prime} \bar{\Psi}_{1}(Q),
\end{aligned}
$$

where $n_{0}^{\prime} \equiv \partial n_{0}\left(Q, \Phi_{0}\right) / \partial \Phi_{0}$. Substituting Eqs. (56) and (57) into Eqs. (53) and (54), we obtain the integral equation for the next-order correction $\bar{\Psi}_{1}$,

$$
\begin{aligned}
\bar{\Psi}_{1}= & \int d Q^{\prime} L\left(Q, Q^{\prime}\right) \bar{\Psi}_{1}\left(Q^{\prime}\right) n_{0}^{\prime}\left(Q^{\prime}\right)+\Phi_{0}+\Phi_{1} \\
& -\left[\eta^{\alpha} Q^{\alpha} \frac{\partial}{\partial Q^{\alpha}}\right] \Phi_{2}+\frac{1}{2}\left[\eta^{\alpha} \eta^{\beta} Q^{\alpha} Q^{\beta} \frac{\partial^{2}}{\partial Q^{\alpha} \partial Q^{\beta}}\right] \Phi_{0},
\end{aligned}
$$

where $\Phi_{1}$ and $\Phi_{2}$ satisfy the equations

$$
\begin{aligned}
\nabla_{\perp}^{2} \Phi_{1}= & -s_{b}\left(\left[Q^{\alpha} \frac{\partial}{\partial Q^{\alpha}}\right]+\frac{1}{2}\left[\eta^{\alpha} \eta^{\beta} Q^{\alpha} Q^{\beta} \frac{\partial^{2}}{\partial Q^{\alpha} \partial Q^{\beta}}\right]\right) \\
& \times n_{0}(Q), \\
\nabla_{\perp}^{2} \Phi_{2}= & -s_{b}\left[\eta^{\alpha} Q^{\alpha} \frac{\partial}{\partial Q^{\alpha}}\right] n_{0}(Q),
\end{aligned}
$$

with the same boundary conditions as the Green's function 
$L(Q, \bar{Q})$. Applying the transverse Laplacian operator $\nabla_{\perp}^{2}$ to Eq. (58), we obtain a differential equation for the correction term $\bar{\Psi}_{1}$ :

$$
\begin{aligned}
{\left[\nabla_{\perp}^{2}+s_{b} n_{0}^{\prime}(Q)\right] \bar{\Psi}_{1}=} & -s_{b}\left(1+\left[Q^{\alpha} \frac{\partial}{\partial Q^{\alpha}}\right] n_{0}(Q)\right. \\
& \left.+\frac{1}{2}\left[\eta^{\alpha} \eta^{\beta} Q^{\alpha} Q^{\beta} \frac{\partial^{2}}{\partial Q^{\alpha} \partial Q^{\beta}}\right]\right) n_{0}(Q) \\
& -\nabla_{\perp}^{2}\left[\eta^{\alpha} Q^{\alpha} \frac{\partial}{\partial Q^{\alpha}}\right] \Phi_{2} \\
& +\frac{1}{2} \nabla_{\perp}^{2}\left[\eta^{\alpha} \eta^{\beta} Q^{\alpha} Q^{\beta} \frac{\partial^{2}}{\partial Q^{\alpha} \partial Q^{\beta}}\right] \Phi_{0} .
\end{aligned}
$$

The boundary condition for the correction term $\bar{\Psi}_{1}$ follows from Eq. (58) and the boundary conditions for $\Phi_{0}, \Phi_{1}$, and $\Phi_{2}$.

It is of particular interest to consider a perfectly conducting cylindrical boundary which is located at a distance $R$ from the beam center. In this case, $L(x, \bar{x})=0$ on the surface $\left(x^{1}\right)^{2}+\left(x^{2}\right)^{2}=R^{2}$. As explained above, the boundary surface in coordinate space $\left(Q^{1}, Q^{2}\right)$ is given by the condition $L(Q, \bar{Q})=0$, and therefore is given by $\left(Q^{1}\right)^{2}+\left(Q^{2}\right)^{2}=R^{2}$. On this surface, $\Phi_{0}=\Phi_{1}=\Phi_{2}=$ 0 . Note, that this surface is not the conductor boundary, which is given by $\left(x^{1}\right)^{2}+\left(x^{2}\right)^{2}=R^{2}$.

It is convenient to introduce the cylindrical coordinates $(r, \theta)$, where $Q^{1}=r \cos (\theta)$ and $Q^{2}=r \sin (\theta)$. It follows from Eqs. (56) that $\Phi_{0}(Q)=\Phi_{0}(r)$ and $n_{0}(Q)=n_{0}(r)$, and Eqs. (56) and (59) become

$$
\begin{aligned}
& \frac{1}{r^{2}} \hat{A}^{2} \Phi_{0}(r)=-s_{b} n_{0}(r), \\
& \frac{1}{r^{2}}\left(\hat{A}^{2}+\frac{\partial^{2}}{\partial \theta^{2}}\right) \Phi_{1}(r)=-\frac{s_{b}}{4}[(\hat{A}+4) \hat{A} \\
& \\
&+\cos (4 \theta)(\hat{A}-2) \hat{A}] n_{0}(r), \\
& \frac{1}{r^{2}}\left(\hat{A}^{2}+\frac{\partial^{2}}{\partial \theta^{2}}\right) \Phi_{2}(r)=-s_{b} \cos (2 \theta) \hat{A} n_{0}(r),
\end{aligned}
$$

where $\hat{A}=r d / d r$. Integrating Eqs. (61) with the appropriate boundary conditions corresponding to $\Phi_{0}(r=R)=$ $\Phi_{1}(r=R)=\Phi_{2}(r=R)=0$, and substituting the results into Eqs. (58) and (60), we obtain equations for the correction term $\bar{\Psi}_{1}$, which can be expressed as $\bar{\Psi}_{1}=p(r)+$ $\cos (4 \theta) q(r)$, where

$$
\begin{aligned}
& \left(\frac{1}{r} \frac{d}{d r} r \frac{d}{d r}+s_{b} n_{0}^{\prime}(r)\right) p(r) \\
& =-s_{b} \frac{8}{R^{4}} \int_{0}^{R} d r r^{3} n_{0}(r), \\
& \left(\frac{1}{r} \frac{d}{d r} r \frac{d}{d r}-\frac{16}{r^{2}}+s_{b} n_{0}^{\prime}(r)\right) q(r) \\
& =-2 s_{b}\left(n_{0}+\frac{4}{r^{2}} \int_{0}^{r} d \bar{r} \bar{r} n_{0}(\bar{r})-\frac{12}{r^{4}} \int_{0}^{r} d \bar{r} \bar{r}^{3} n_{0}(\bar{r})\right),
\end{aligned}
$$

with boundary conditions

$$
\begin{aligned}
& p(R)=-\frac{2 s_{b}}{R^{2}} \int_{0}^{R} d r r^{3} n_{0}(r), \\
& q(R)=-s_{b}\left(\frac{2}{R^{2}} \int_{0}^{R} d \bar{r} \bar{r}^{3} n_{0}(\bar{r})-\frac{1}{2} \int_{0}^{R} d \bar{r} \bar{r} n_{0}(\bar{r})\right) .
\end{aligned}
$$

Note from Eqs. (62) and (63) that $p(R) \neq 0$ and $q(R) \neq 0$, and therefore $\bar{\Psi} \neq 0$ on the boundary $r=R$. This is because for a quadrupole channel the boundary $r=R$ is not a real conductor surface, but a surface that oscillates around the conductor surface with an amplitude $\delta R / R \sim$ $\epsilon\left[\left\langle v^{2}\right\rangle\right]^{1 / 2}$.

As a specific example, we consider a beam with constant average density profile inside the average radius $r=a$, located inside a perfectly conducting pipe at $r=R$, i.e.,

$$
n_{0}(r)= \begin{cases}\bar{n}_{0}, & r<a, \\ 0, & a<r \leq R .\end{cases}
$$

This beam density profile is produced self-consistently by the Kapchinskij-Vladimirskij distribution $G(K)=$ $\left(\bar{n}_{0} / 2 \pi\right) \delta\left(K-K_{0}\right)[5,19]$. For this choice of density profile, the potential $\Phi_{0}(r)$ takes the form

$$
\Phi_{0}(r)= \begin{cases}-s_{b} \bar{n}_{0} \frac{r^{2}}{4}+s_{b} \bar{n}_{0} \frac{a^{2}}{4}\left(1-2 \ln \frac{a}{R}\right), & r<a, \\ -s_{b} \bar{n}_{0} \frac{a^{2}}{2} \ln \frac{r}{R}, & a<r \leq R .\end{cases}
$$

It follows from Eqs. (64) and (65) that $s_{b} n_{0}^{\prime}(r)=$ $-2 \bar{s} /(1-\bar{s}) \delta(r-a) / a . \quad$ Here, $\quad \bar{s} \equiv \omega_{p}^{2} / 2 \omega_{s f}^{2} \equiv$ $s_{b} \bar{n}_{0} /\left[2\left\langle\left(\kappa^{(1)}\right)^{2}\right\rangle\right]$ [5]. The solution to Eqs. (62) and (63) gives the correction term $\bar{\Psi}_{1}=p(r)+\cos (4 \theta) q(r)$, where the functions $p(r)$ and $q(r)$ are given by

$$
p(r)=-s_{b} \bar{n}_{0} \frac{a^{2}}{2}\left(\frac{a}{R}\right)^{4} \begin{cases}\left(\frac{r}{a}\right)^{2}+\frac{[2 \bar{s} /(1-\bar{s})] \ln (a / R)}{1-[2 \bar{s} /(1-\bar{s})] \ln (a / R)}, & r<a, \\ \left(\frac{r}{a}\right)^{2}+\frac{[2 \bar{s} /(1-\bar{s})] \ln (r / R)}{1-[2 \bar{s} /(1-\bar{s})] \ln (a / R)}, & a<r \leq R,\end{cases}
$$

and

$$
q(r)=s_{b} \bar{n}_{0} \frac{a^{2}}{4} \begin{cases}-\left(\frac{r}{a}\right)^{4}\left(\frac{a}{R}\right)^{8} \frac{1}{1+\{\bar{s} /[4(1-\bar{s})]\}\left[1-(a / R)^{8}\right]}, & r<a, \\ 1-2\left(\frac{a}{r}\right)^{2}+\left(\frac{a}{r}\right)^{4}\left[1-\left(\frac{r}{R}\right)^{8}\right] \frac{1+\{\bar{s} /[4(1-\bar{s})]\}}{1+\{\hat{s} /[4(1-\bar{s})]\}\left[1-(a / R)^{8}\right]}, & a<r \leq R .\end{cases}
$$


The corrections to the electric field corresponding to the correction to the potential given by Eqs. (66) and (67) contain two parts. One part is the correction which remains finite as $R \rightarrow \infty$ keeping $r$ finite. This part of the field correction is due to the average effects of the beam density oscillations, and is a decreasing function of $r$ outside the beam. The other part is the correction which vanishes as $R \rightarrow \infty$ keeping $r$ finite. This part is due to the average field created inside the region surrounded by the boundary by the oscillating charges on the boundary induced by the oscillating beam distribution. It exists because the induced distribution of charges on the boundary is not cylindrically symmetric for a beam in a quadrupole channel. This part is an increasing function of $r$.

Corrections to the self-field potential leads to the correction to the average beam radius given by

$$
r_{b}(\theta)=a\left(1-\epsilon^{2}\left\langle v^{2}\right\rangle \frac{2 \bar{s}}{(1-\bar{s})} \frac{\bar{\Psi}_{1}(a, \theta)}{s_{b} \bar{n}_{0} a^{2}}\right),
$$

which now becomes weakly dependent on the angle $\theta$ according to

$$
\begin{aligned}
r_{b}(\theta)= & a\left\{1+\epsilon^{2}\left\langle v^{2}\right\rangle \frac{\bar{s}}{(1-\bar{s})}\left(\frac{(a / R)^{4}}{1-\frac{2 \bar{s}}{1-\bar{s}} \ln \frac{a}{R}}\right.\right. \\
& \left.\left.+\frac{\cos (4 \theta)}{2} \frac{(a / R)^{8}}{1+\frac{\bar{s}}{4(1-\bar{s})}\left[1-\left(\frac{a}{R}\right)^{8}\right]}\right)\right\} .
\end{aligned}
$$

As expected, the corrections to the self-field potential and the beam density [Eq. (57)] inside the beam, and corrections to the beam radius are zero when $R \rightarrow \infty$, and the total self-field potential inside the beam is given by Eq. (65), which is what one would expect for a Kapchinskij-Vladimirskij distribution in free space $(R \rightarrow$ $\infty$ ) which generates the constant beam density given by Eq. (64). When the conducting boundary is present ( $R$ is finite), the image charge oscillations produce additional contributions to the average self-field potential inside the beam, which lead to the octupole correction to the average beam radius [Eqs. (68) and (69)].

\section{DISCUSSION OF RESULTS AND CONCLUSIONS}

In conclusion, in this paper we have studied the nonlinear transverse dynamics of an intense charged particle beam propagating inside a periodic focusing-defocusing lattice with period $S$ and characteristic focusing lattice strength $\kappa_{0}$. For this system, we have identified a small expansion parameter $\bar{\epsilon} \sim \sigma_{v} / 2 \pi$, where $\sigma_{v}$ is the vacuum phase advance [5]. Using a consistent normalization, we have developed a perturbative canonical Hamiltonian transformation method in Eqs. (29)-(31), which we use to transform away the fast particle oscillations with lattice period $S$, and obtain the average Hamiltonian accurate to order $\bar{\epsilon}^{3}$ [Eq. (36)] and the canonical transformation accurate to the order $\bar{\epsilon}^{2}$ [Eqs. (32)-(35)] for arbitrary periodic focusing lattice. The normalization used in the present paper is different from the one used by previous authors [24-26] in that we regard the oscillation component of the particle momentum to be of the same order as the average component. This normalization is confirmed by the simulations and the final expression for the average Hamiltonian in Eq. (36), where all terms are of the same order. Because the average particle motion is on surfaces of constant average energy $K=$ const, in general we obtain $P \sim Q \sim 1$ from the average Hamiltonian in Eq. (36), which is what was assumed initially. The Hamiltonian obtained in Refs. [24-26] multiplies the average external potential by the square of the small expansion parameter $(\sqrt{\bar{\epsilon}})^{2}$, which implies that $Q \sim P / \sqrt{\bar{\epsilon}}$, which in turn is inconsistent with the ordering made in the derivation $Q \sim$ $P \sim 1$ in Refs. [24-26].

It should also be pointed out that the iterative procedure used in obtaining the canonical transformation in Eqs. (29)-(31) is very explicit, and for any given lattice can be easily programmed into a software package such as MATHEMATICA [32] to obtain the canonical transformation valid to arbitrary order.

Using the average Hamiltonian, we obtained the expression for the vacuum phase advance [Eq. (37)] accurate to third order in the small parameter $\bar{\epsilon} \sim \sigma_{v} / 2 \pi$. Because of the cancellation of some terms for lattices satisfying the symmetry condition $\kappa(s)=-\kappa(s+S / 2)$, the results for the average Hamiltonian and the vacuum phase advance are accurate up to fourth order in the small parameter $\bar{\epsilon}$. The results obtained using this method are consistent with previous results obtained to third order in the small parameter $\sqrt{\bar{\epsilon}}$ in Refs. [24,25]. In this paper, we have extended the perturbative treatment to third order (for the average Hamiltonian) in $\bar{\epsilon} \sim \sigma_{v} / 2 \pi$ (or to the sixth order in the small parameter $\sqrt{\bar{\epsilon}}$ used in previous treatments) by performing a consistent normalization, and by avoiding the unnecessary calculation of the generating function as a function of a mixed set of canonical variables. For a specific choice of distribution function $G(K)$, Eq. (39) can be solved to determine the new time-independent Hamiltonian $K$. The corrections to the new Hamiltonian are of two kinds. The corrections to the kinetic energy term and the applied average potential term give corrections to the average frequency of the particle motion in the external oscillating field. We have determined these corrections for both sinusoidal and step-function lattices in Sec. IV. The corrections allow us to extend the average formulaic results to larger vacuum phase advances approaching $\sigma_{v} \sim 90^{\circ}$ with accuracies to within several percent. The use of the expressions for the canonical transformations [Eqs. (32)(36)] allows an accurate representation of the original highly oscillatory trajectories for much longer periods of time than previously possible. In fact, the phase error 
accumulates with time at a rate proportional to $\bar{\epsilon}^{5} s$ [see Fig. 1].

The average self-field potential was determined in Sec. V. We used the representation of the average self-field potential given by Eq. (51) which uses the Green's function for the original unaveraged configuration with arbitrary boundary conditions to derive the differential equations for the average self-field potential [Eqs. (56)-(60)]. In this representation, the average potential is the average of the potential produced by the beam and by the charges that are induced on the conducting boundary. The average selffield potential $\bar{\Psi}[$ Eq. (57)] can be represented as a sum of a zero-order smooth-focusing part $\Phi_{0}$, which satisfies the Poisson equation (56), plus the next-order correction $\epsilon^{2}\left\langle\left(\kappa^{(2)}\right)^{2}\right\rangle \bar{\Psi}_{1} \sim\left(\sigma_{v} / 2 \pi\right)^{2}$, where the function $\bar{\Psi}_{1}$ satisfies the inhomogeneous Helmholz-type equation in Eq. (60). For the particular case of a cylindrical, perfectly conducting boundary with radius $R$, the correction term $\bar{\Psi}_{1}$ can be expressed in cylindrical coordinates $(r, \theta)$ as $\bar{\Psi}_{1}=p(r)+$ $\cos (4 \theta) q(r)$, where the functions $p(r)$ and $q(r)$ satisfy Eq. (62), with the boundary conditions given by Eq. (63). Note that the average self-field potential acquires an octupole component, which results in the average motion of some beam particles being nonintegrable and their trajectories becoming chaotic. This chaotic behavior of some of the beam particles may significantly change the nature of the Landau damping (or growth) of collective excitations supported by the beam. As a particular example, in Sec. V we studied an intense charged particle beam with Kapchinskij-Vladimirskij distribution [5] inside a perfectly conducting pipe with wall radius $R$. It was shown that the correction to the average self-field potential inside the beam is zero when the boundary is removed to $R \rightarrow \infty$. When the boundary location is finite ( $R$ is finite), the average motion of the image charges induces nonzero corrections to the average self-field potential inside the beam [Eqs. (66) and (67)], which results in the average beam radius acquiring the octupole component in Eqs. (68) and (69).

Finally, an interesting application of the average selffield equations (53) and (54) is for the case of very intense beams, when the zero-order smooth-focusing part $\Phi_{0}$ of the average self-field potential is compensated by the average applied focusing potential. In this case the secondorder corrections to the average self-field potential $\bar{\Psi}$ in Eq. (53) could become the dominant contributions to the total self-field potential, and determine the collective dynamics of the beam particles. Such dynamics would be similar to the particle dynamics in a strongly magnetized quasineutral plasma using a "gyrokinetic" description [33].

\section{ACKNOWLEDGMENTS}

This research was supported by the U.S. Department of Energy.
[1] B. G. Logan, F. M. Bieniosek, C. M. Celata, J. Coleman, W. Greenway, E. Henestroza, J. W. Kwan, E. P. Lee, M. Leitner, P. K. Roy, P. A. Seidl, J.-L. Vay, W. L. Waldron, S. S. Yu, J. J. Barnard, R. H. Cohen, A. Friedman, D. P. Grote, M. Kireeff Covo, A. W. Molvik, S. M. Lund, W. R. Meier, W. Sharp, R. C. Davidson, P. C. Efthimion, E. P. Gilson, L. Grisham, I. D. Kaganovich, H. Qin, A. B. Sefkow, E. A. Startsev, D. Welch, and C. Olson, Nucl. Instrum. Methods Phys. Res., Sect. A 577, 1 (2007).

[2] R. C. Davidson, Physics of Non-Neutral Plasmas (World Scientific, Singapore, 2001), and references therein.

[3] E. D. Courant, M. S. Livingstone, and H. S. Snyder, Phys. Rev. 88, 1190 (1952).

[4] E. D. Courant and H. S. Snyder, Ann. Phys. (N.Y.) 3, 1 (1958).

[5] R. C. Davidson and H. Qin, Physics of Intense Charged Particle Beams in High Energy Accelerators (World Scientific, Singapore, 2001), and references therein.

[6] M. Reiser, Theory and Design of Charged Particle Beams (John Wiley \& Sons, Inc., New York, 1994).

[7] A. W. Chao, Physics of Collective Beam Instabilities in High Energy Accelerators (John Wiley \& Sons, Inc., New York, 1993).

[8] R. C. Davidson, M. Dorf, I. Kaganovich, H. Qin, E. A. Startsev, and S. M. Lund, Nucl. Instrum. Methods Phys. Res., Sect. A 606, 11 (2009).

[9] H. Qin, R. C. Davidson, and E. A. Startsev, Nucl. Instrum. Methods Phys. Res., Sect. A 606, 37 (2009).

[10] E. A. Startsev, R. C. Davidson, and H. Qin, Phys. Plasmas 14, 056705 (2007).

[11] M. Dorf, R. C. Davidson, and E. A. Startsev, Phys. Rev. ST Accel. Beams 9, 034202 (2006).

[12] E. A. Startsev, R. C. Davidson, and H. Qin, Phys. Rev. ST Accel. Beams 8, 124201 (2005).

[13] E. A. Startsev, R. C. Davidson, and H. Qin, Nucl. Instrum. Methods Phys. Res., Sect. A 544, 125 (2005).

[14] E. A. Startsev and R. C. Davidson, Phys. Plasmas 10, 4829 (2003).

[15] H. Qin, E. A. Startsev, and R. C. Davidson, Phys. Rev. ST Accel. Beams 6, 014401 (2003).

[16] E. A. Startsev, R. C. Davidson, and H. Qin, Phys. Rev. ST Accel. Beams 6, 084401 (2003).

[17] E. A. Startsev, R. C. Davidson, and H. Qin, Laser Part. Beams 20, 585 (2002).

[18] E. A. Startsev, R. C. Davidson, and H. Qin, Phys. Plasmas 9, 3138 (2002).

[19] I. M. Kapchinskij and V. V. Vladimirskij, in Proceedings of the International Conference on High Energy Accelerators and Instrumentation (CERN Scientific Information Service, Geneva, 1959), p. 274.

[20] E. A. Startsev and S. M. Lund, Phys. Plasmas 15, 043101 (2008).

[21] E. A. Startsev and R. C. Davidson, New J. Phys. 6, 141 (2004).

[22] R. C. Davidson and E. A. Startsev, Phys. Rev. ST Accel. Beams 7, 024401 (2004).

[23] E. A. Startsev and R. C. Davidson, Phys. Rev. ST Accel. Beams 6, 044401 (2003).

[24] P. J. Channell, Phys. Plasmas 6, 982 (1999).

[25] R. C. Davidson, H. Qin, and P. J. Channell, Phys. Rev. ST Accel. Beams 2, 074401 (1999); 3, 029901 (2000). 
[26] S. I. Tzenov and R.C. Davidson, Phys. Rev. ST Accel. Beams 5, 021001 (2002).

[27] H. Goldstein, Classical Mechanics (Addison-Wesley, Reading, 1980), 2nd ed.

[28] L.D. Landau and E. M. Lifshitz, Classical Mechanics (Pergamon, New York, 1991).

[29] V.I. Arnold, Mathematical Methods of Classical Mechanics (Springer-Verlag, New York, 1989), 2nd ed.

[30] J. E. Marsden and T. Ratiu, Introduction to Mechanics and
Symmetry (Springer-Verlag, New York, 1994).

[31] R.Z. Sagdeev, D. A. Usikov, and G. M. Zaslavsky, Nonlinear Physics: From the Pendulum to Turbulence and Chaos (Harwood Academic Publishers, New York, 1988).

[32] Stephen Wolfram, The Mathematica Book (Cambridge University Press, New York, 1999), 4th ed.

[33] W. W. Lee, Phys. Fluids 26, 556 (1983). 